\title{
The Wellington microlensing modelling programme
}

\author{
Denis J. Sullivan*, Aarno Korpela and Paul Chote \\ School of Chemical \& Physical Sciences, \\ Victoria University of Wellington, \\ P O Box 600, Wellington, New Zealand. \\ E-mail: denis.sullivan@vuw.ac.nz, a.korpela@niwa.co.nz, \\ pauldchote. net
}

\begin{abstract}
We provide a brief description of the microlensing modelling activities being undertaken in Wellington, New Zealand. Largely as part of a recently completed PhD project, we have developed a flexible software suite to model complex gravitational microlensing events. Written using $\mathrm{C}++$, the code implements the inverse ray-tracing technique to model the light curves corresponding to multiple point mass lensing events. The software has been developed to run on parallel processing systems, as we have at our disposal a number of computing grids. These include a Sun Grid Engine that has access to about 100 nodes running a flavour of BSD Unix, a grid of about 1000 Windows machines connected using the Condor distributed computing software, and an IBM Blue Gene system (located at the University of Canterbury in Christchurch NZ. The modelling of a number of recent events of topical interest is presented, specifically MOA 2002BLG-42, OGLE 2007-BLG-349 and OGLE 2006-BLG-109.
\end{abstract}

The Manchester Microlensing Conference: The 12th International Conference and ANGLES Microlensing Workshop

January 21-25, 2008

Manchester, $U K$

\footnotetext{
* Speaker.
} 


\section{Introduction}

The use of the gravitational microlensing technique to search for extrasolar planets has become both a successful and topical activity in the past few years. There are three essential components to this work. First, interesting (mostly high-magnification) microlensing events need to be detected and alerted. The two currently operating survey groups, MOA [1] and OGLE [2], now alert something approaching 1000 Galactic bulge events per year combined. Second, intensive follow-up observations are required around peak magnification times in order to detect and monitor any departures from the predictable point-mass lensing changes. Follow up groups such as MicroFUN [3] and PLANET [4] combine successfully with MOA and OGLE to obtain these data. And, third, the necessary software and computing resources are require in order to successfully model the resulting complex events. A number of groups undertake this work, but our experience has been that there is a significant effort involved in accumulating and developing the required resources. The following provides an overview of our activities along with several modelling examples.

\section{Some relevant microlensing theory}

It is well established that an observer viewing a luminous point-like source 'sees' two distorted images when an intervening stellar mass passes very close to the direct line of sight causing so-called gravitational microlensing. The positions of the two images (and the resulting flux magnification if the images can't be resolved) can be readily obtained from the simple scalar equation relating the actual source position $\left(\eta_{E}\right)$ to the two image positions $\left(\xi_{E}\right)$ :

$$
\eta_{E}=\xi_{E}-\frac{1}{\xi_{E}}
$$

This expression assumes the thin lens approximation and the two quantities $\eta_{E}$ and $\xi_{E}$ are projected distances in the source plane in units of the projected angular radius, $D_{S} \theta_{E}$, on to the same plane, where the Einstein ring angle is given by

$$
\theta_{E}=\left[\frac{2 R_{g}\left(D_{S}-D_{L}\right)}{D_{S} D_{L}}\right]^{\frac{1}{2}} \quad \text { with } \quad R_{g}=\frac{2 G M}{c^{2}}
$$

Note that standard notation has been employed, with $D_{L}$ and $D_{S}$ being the observer to lens and source distances, respectively, and $R_{g}$ is the Schwarzschild radius for the lensing mass. It is clear that for Galactic distances and stellar masses, $\theta_{E}$ is very small (milliarcseconds) and the the two images therefore cannot be resolved. But the changing magnification due to the relative lens component motion has been observed (many thousands of times!).

Adding only one additional point mass to the lensing configuration changes things substantially. This is rather surprising, and the full complexity of the changes are not immediately evident from the form of generalised equation describing the lens system. Again using the thin lens approximation this becomes:

$$
\vec{\eta}_{E}=\vec{\xi}_{E}-\left(\frac{M_{1}}{M_{1}+M_{2}}\right) \frac{\vec{\xi}_{E}}{\left|\vec{\xi}_{E}\right|^{2}}-\left(\frac{M_{2}}{M_{1}+M_{2}}\right) \frac{\vec{\xi}_{E}-\vec{\rho}_{E}}{\left|\vec{\xi}_{E}-\vec{\rho}_{E}\right|^{2}}
$$




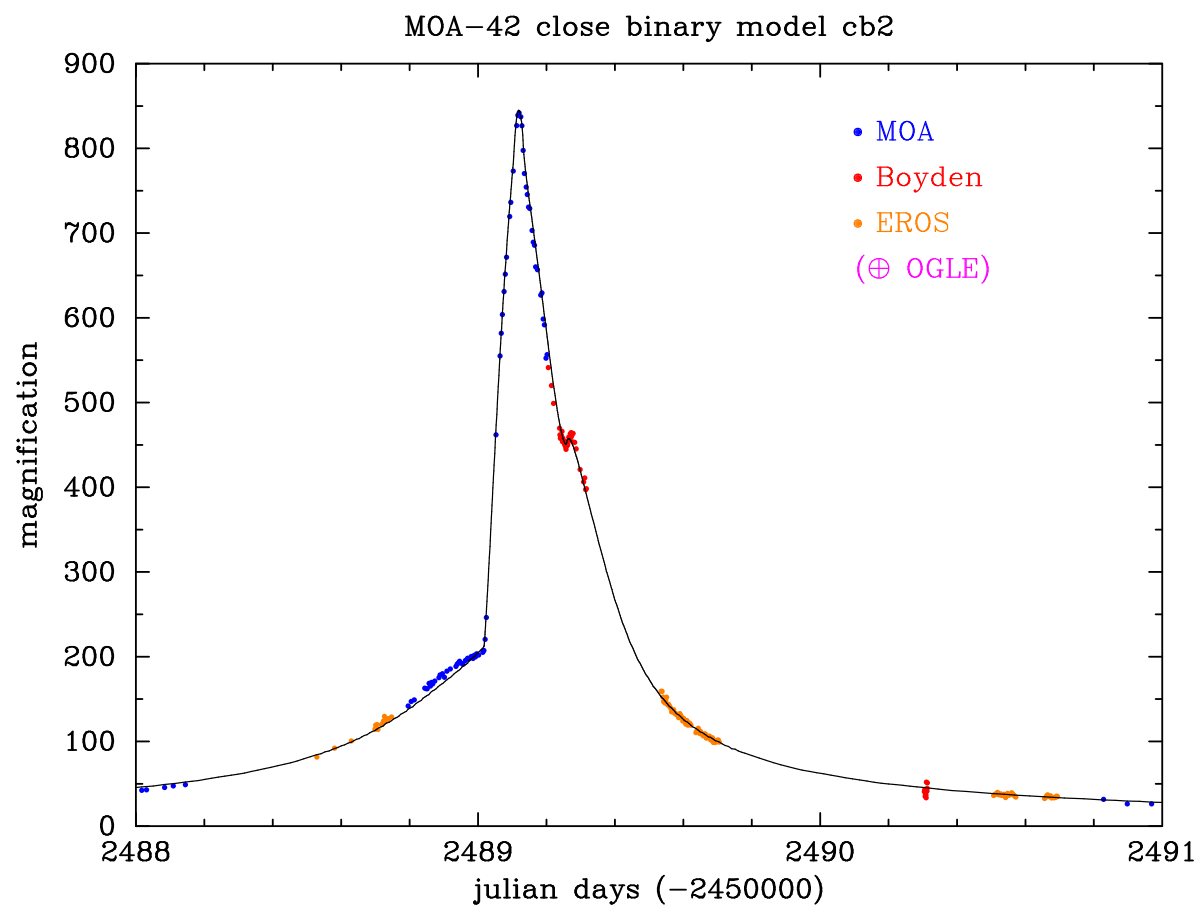

Figure 1: The event MOA 2002-BLG-42 modelled using the cb2 configuration depicted in the Fig. 2 caustic curve plots.

The source $\left(\vec{\eta}_{E}\right)$ and image positions $\left(\vec{\xi}_{E}\right)$ now require specification by projected vector displacements (in units of the Einstein ring radius here) in the source plane along with the separation, $\vec{\rho}_{E}$, between the two lensing masses. The angular Einstein ring value used in this formula corresponds to the total mass, $M_{1}+M_{2}$, and with a view to modelling star-planet lens systems the origin of the coordinate system in the source plane corresponds to the projected position of the primary (stellar) lensing mass.

Of particular relevance for modelling binary lensing events is that this seemingly straight forward extension to the single mass lens case cannot be solved analytically. Numerical techniques are required to deduce the magnification curves and other quantities such as the caustic curves. The caustic curves are the multi-lens analogue of the point source lens perfect alignment single position, and correspond to all the (point) source positions that result in (formally) infinite magnification, and they signal a change in the number of images when the source track crosses them. Caustic curve plots provide a useful insight into the predicted magnification changes.

A straight forward, but computing intensive method, to determine magnification values for binary lens configurations is inverse ray tracing $[5,6]$. In this approach, various positions in the source plane are exhaustively checked using the above equation to see if they are consistent with a region in this plane corresponding to a given source position. When all the image positions and their areas have been mapped out, the magnification can be estimated from the image/source area ratio. Or, a magnification map can be created by recording the complete mapping of possible image positions on to the various source positions governed by the above equation. The speed of modern computers makes these methods practicable. 


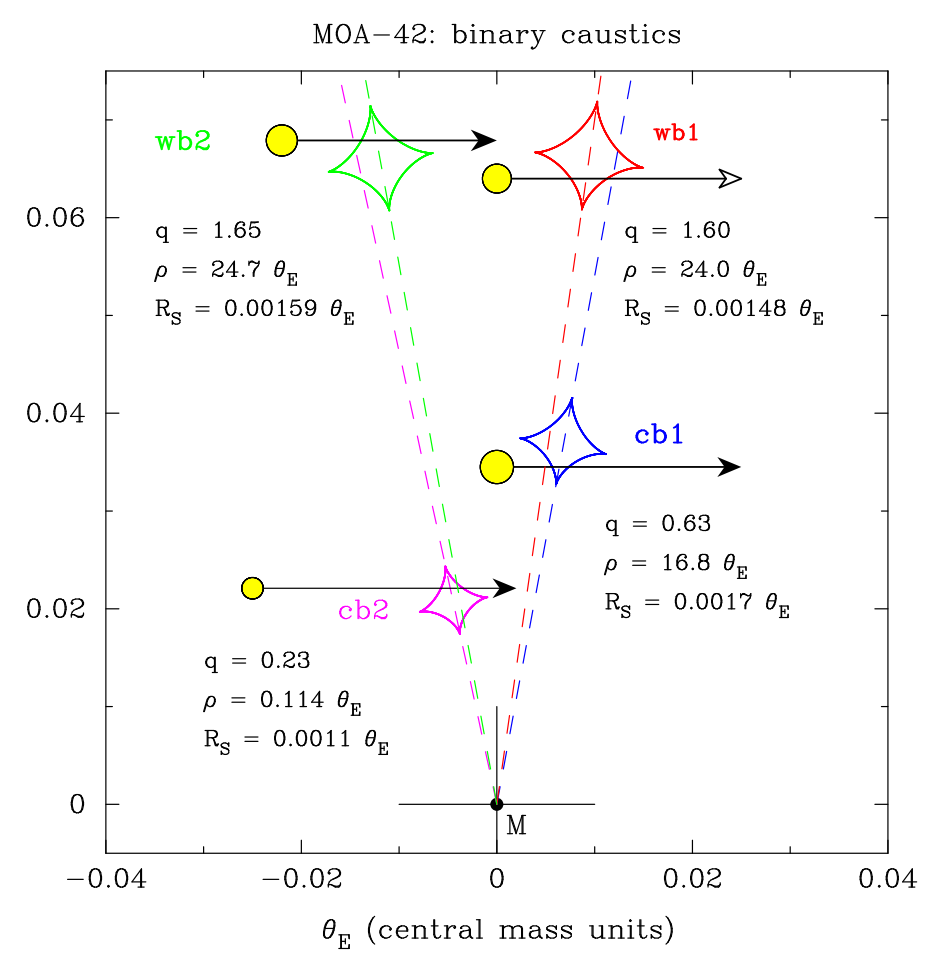

Figure 2: The caustic curves for four of the binary model fits to the event MOA 2002-BLG-42. The fit corresonding to cb1 is poor, while the other 3 fits are reasonable with wb2 being the best. However, there is a discrepancy that may indicate the presence of a third body. Note the the two models wb1 and wb2 have mass ratios $(q)$ greater than $1, \rho$ is the lens component separation and $\mathrm{R}_{S}$ is the source radius.

One advantage of this approach is that it is readily extended to include three or more lensing masses. In addition, finite source sizes are naturally incorporated, and limb-darkening effects also can be readily included.

\section{Wellington software and hardware}

As part of a PhD project [7] a programming suite that utilises the inverse ray tracing technique has been developed in order to model binary lensing events. Coding conforms to the ANSI C++ standard and the programs are structured using object class properties and methods. The top-level modelling program (MLmodel) creates magnification maps for each parameter set and navigates around the data versus model $\chi^{2}$ hypersurface in order to find the best model parameters, using a number of techniques, including a Monte Carlo Markov Chain (MCMC) stochastic algorithm. Appropriate magnification maps are created as needed and maximum use is made of parallel processing by using different computing grid nodes to perform various tasks.

The program suite also includes the package MLview, which provides an interactive display of light curves, caustics and critical curves for multiple point mass lens systems. This capability has been found to be very useful for finding an appropriate starting set of parameters for MLmodel. The caustic and critical curves for a configuration are determined using the program gravlens [8]. 


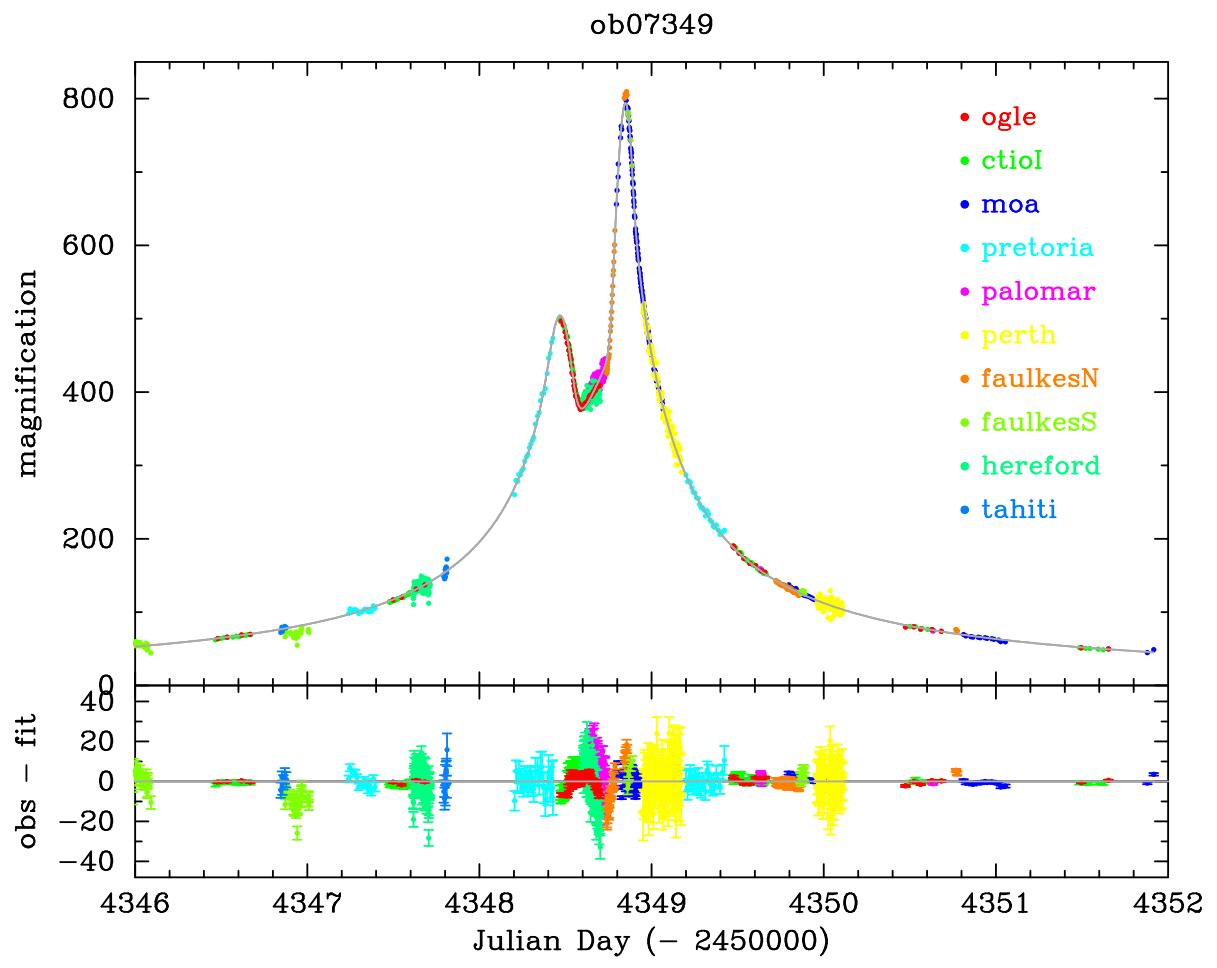

Figure 3: The microlensing event OGLE 2007-BLG-349 with a best binary lens fit.

We have access to three parallel processing systems: (1) a network of approximately 100 PCs running a flavour of BSD Unix, with the processing resources coordinated using the Sun Grid Engine (SGE) software, (2) approximately 1000 PCs running Windows software with coordination provided by the Condor [9] grid computing software, and (3) an IBM p575/Blue Gene system at the University of Canterbury in Christchurch, available via remote login. Most of our modelling work to date has been carried out on the SGE system, but we are migrating work to the VUW Condor system as well as investigating the relative performance of the IBM system.

\section{Modelling of specific events}

Figs. 1 to 7 depict some of the results from modelling three interesting events: MOA 2002BLG-42, OGLE 2007-BLG-349 and OGLE 2006-BLG-109.

The first event derives from 2002 when the MOA collaboration detected and alerted several binary lens high magnifcation events. One of these (MOA 2002-BLG-33) resulted in a flux magnification of nearly 500, and given that good coverage was obtained with observations from other sites, modelling of the event provided an effective probe of the source star atmosphere and stellar profile $[10,11]$. MOA-42 is another binary event that occurred somewhat later in the same season and featured a magnification of more than 800 according to one of the model fits. We did obtain follow-up observations from two other sites (Boyden, South Africa and EROS, Chile) that together with MOA data provided a reasonable characterisation of the event, but our analysis has not yet been formally published. In Figs. 1 and 2 we illustrate some of our modelling results. 


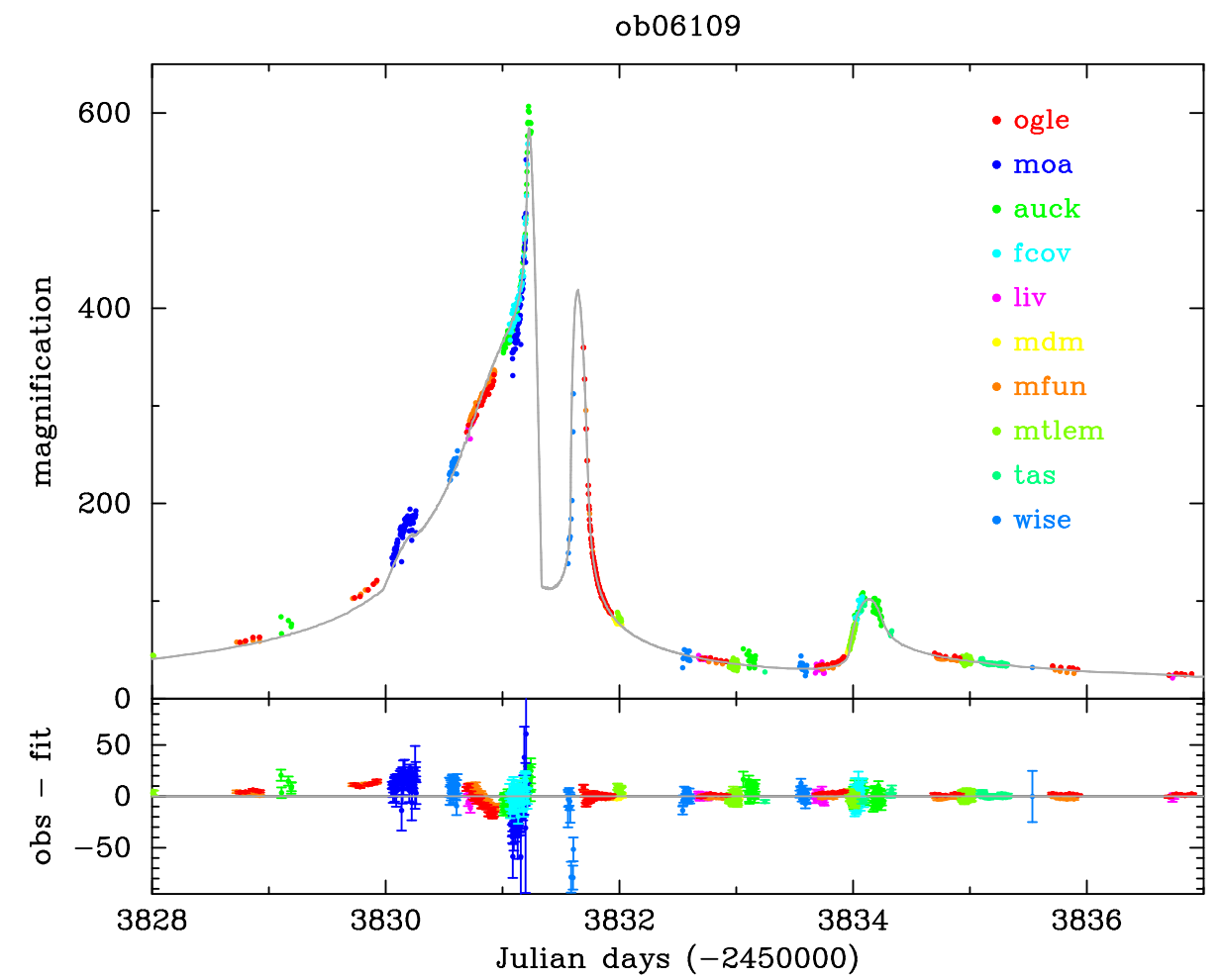

Figure 4: The intriguing event OGLE 2006-BLG-109 requires three lensing masses to reproduce the topology of the observed light curve. The model fit graphed here does not include lens component orbital motion and microlensing parallax effects - see [13] for an optimum model fit.
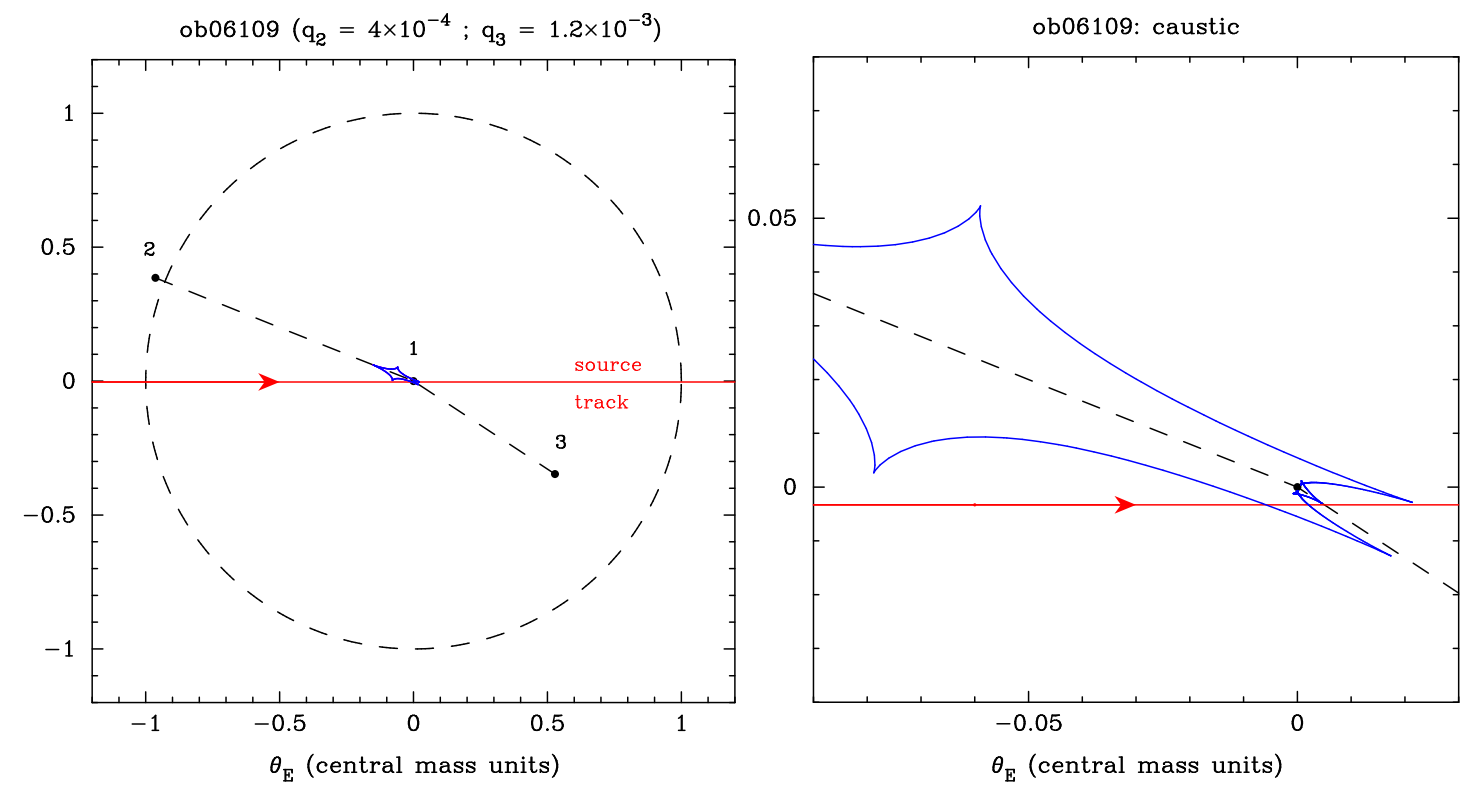

Figure 5: Caustic curve and source track geometry for the OGLE 2006-BLG-109 event model depicted in Fig. 4, showing the lens component positions relative to the Einstein ring radius (left) and an expanded picture of the central caustic. 

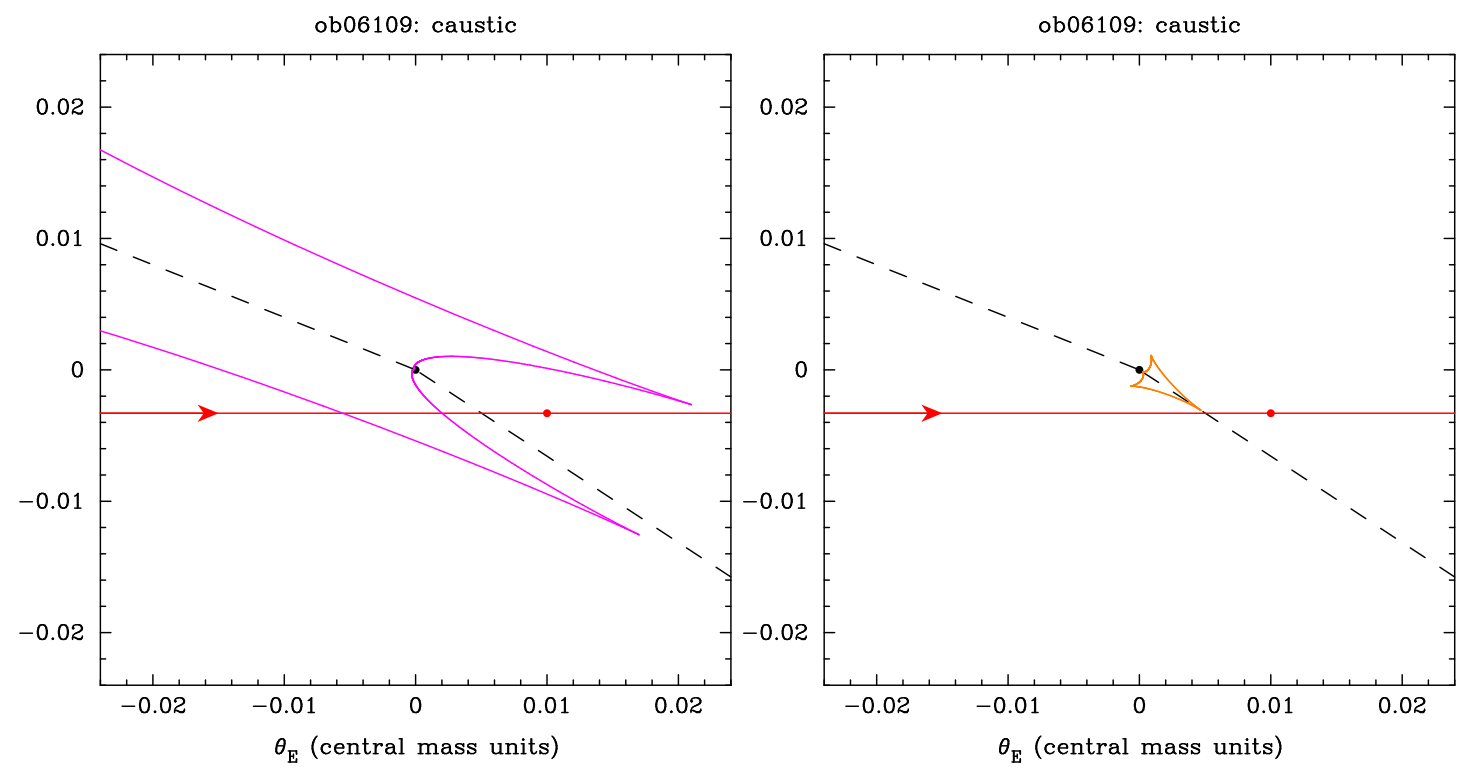

Figure 6: Caustic curve geometry for the OGLE 2006-BLG-109 event showing the individual caustic contributions from lensing mass 2 (left) and lensing mass 3 (right).
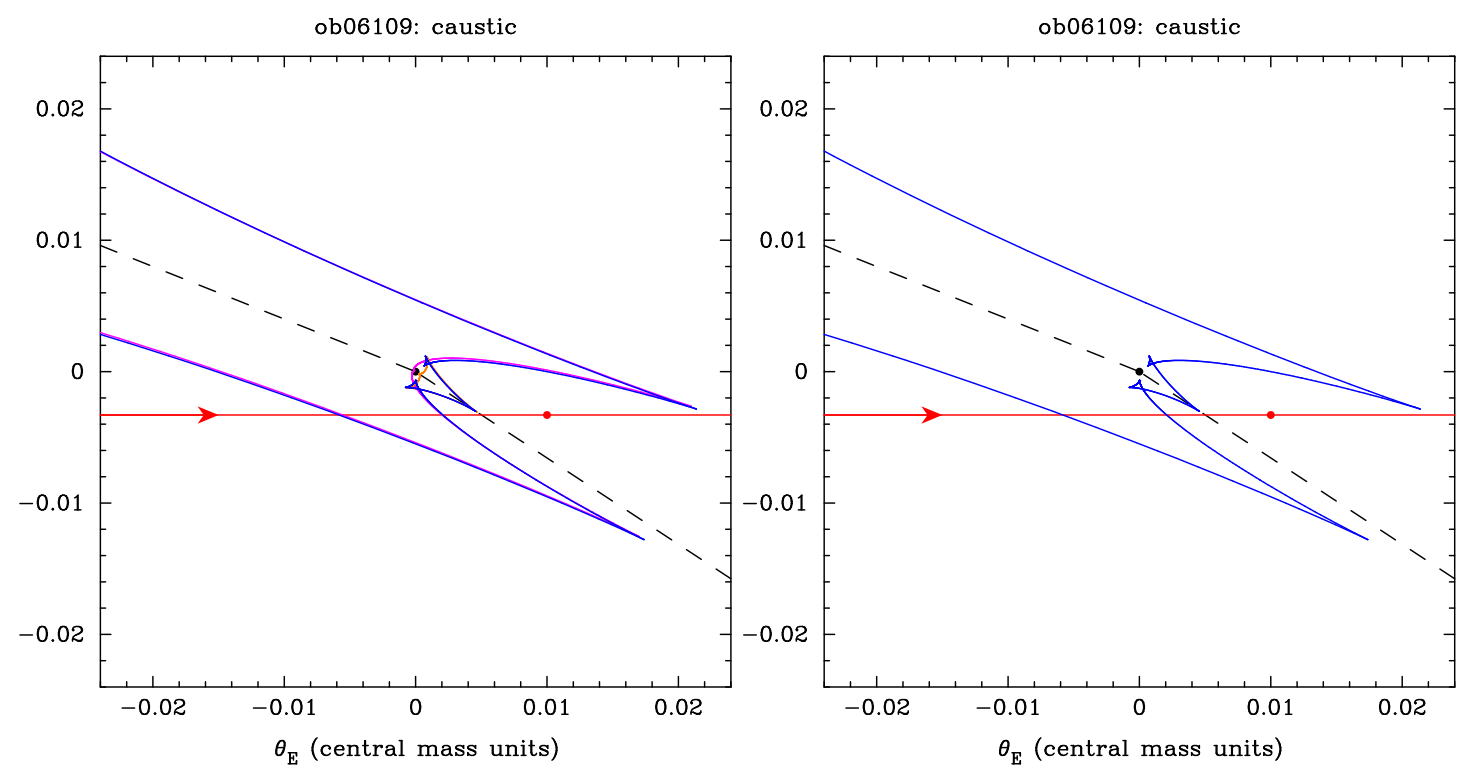

Figure 7: Caustic curve geometry for the OGLE 2006-BLG-109 event comparing a superposition of the separate binary caustics (left) with the combined three-mass caustic (right). 
The event OGLE 2007-BLG-349 is a recent well-covered binary event, but with some suggestion of a third mass required to obtain an optimum fit [12]. Fig. 3 depicts our best binary lens fit to the data.

OGLE 2006-BLG-109 is the fascinating event recently published [13] that requires a threemass lens for optimum modelling. In Figs. 4 to 7 we provide the results of our approximate modelling that focusses on the topology of the three-mass system. Complete modelling of this event requires accounting for Earth's orbital motion during the event (microlensing parallax) and also the orbital motion of the lens outer planet [13]. The figures presented here are designed to reveal the interesting fact that the relevant caustic curve structure for the three-mass system is close to a superposition of the individual two mass systems (Figs. 6 and 7).

\section{Acknowledgments}

We thank the computer scientists at Victoria University of Wellington for establishing and maintaining the computing grids that are a key part of this work. We also acknowledge the Marsden Fund of New Zealand for their financial support for the MOA project.

\section{References}

[1] The Japan/NZ Microlensing Observations in Astrophysics collaboration (MOA), http://www.phys.canterbury.ac.nz/moa/

[2] The Optical Gravitational Lensing Experiment (OGLE), http://www.astrow.edu.pl/ ogle/

[3] Microlensing Follow-Up Network (MicroFUN), http://www.astronomy.ohio-state.edu/ microfun/

[4] Probing Lensing Anomalies NETwork (PLANET), http://planet.iap.fr/

[5] P. Schneider and A. Weiss, The two-point-mass lens: detailed investigation of a special asymmetric gravitational lens, Astronomy \& Astrophysics 164237 (1986)

[6] J. Wambsganss, Discovering Galactic planets by gravitational microlensing: magnification patterns and light curves Mon. Not R. Astron. Soc. 284172 (1997)

[7] A. Korpela, Modelling complex gravitational microlensing events, $\mathrm{PhD}$ thesis, Victoria University of Wellington (2007)

[8] C. Keeton, http://redfive.rutgers.edu/ keeton/gravlens

[9] Condor High Throughput Computing (HTC), http://www.cs.wisc.edu/condor/

[10] F. Abe, D. P. Bennett, I. A. Bond et al. (the MOA collaboration), Probing the atmosphere of a solar-like star by galactic microlensing at high magnification, Astronomy \& Astrophysics 411 L493 (2003)

[11] N. J. Rattenbury, F. Abe, D. P. Bennett et al. (the MOA collaboration), Determination of stellar shape in microlensing event MOA 2002-BLG-33 Astronomy \& Astrophysics 439645 (2005)

[12] S. Dong (dong@astronomy.ohio-state.edu), private communication

[13] B. S. Gaudi, D. P. Bennett, A. Udalski et al., Discovery of a Jupiter/Saturn analog with gravitational microlensing, Science 319927 (2008) 